\title{
TRAUMATIC SHOCK. II. THE PREPARATION OF CYSTINE, METHIONINE, AND HOMOCYSTINE CONTAINING RADIOACTIVE SULFUR
}

\author{
By ARNOLD M. SELIGMAN, ALEXANDER M. RUTENBURG, AND HENRY BANKS 1 \\ (From the Surgical Research Department of the Beth Israel Hospital and the Department of \\ Surgery, Harvard Medical School, Boston)
}

(Received for publication November 12, 1942)

In order to prepare, from radioactive sulfur, the sulfur-containing amino acids of a high order of specific activity for biological experiments such as those described in the foregoing publication, it was necessary (owing to the cost of radioactive sulfur) to investigate the efficiency of utilization of small amounts of sulfur. The synthetic methods utilized are not novel, but are described below because of the data obtained on yields in numerous small scale preparations. Since present methods of preparing radioactive sulfur from neutron bombardment of carbon tetrachloride involve its isolation and purification as $\mathrm{BaSO}_{4}(1)$, the schema of synthesis used for the preparation of three sulfur containing amino acids begins with barium sulfate (Figure 1).

The synthesis of radioactive methionine (VI) from radioactive sulfur ( 0.05 moles) was reported by Tarver and Schmidt (2), using a modification of the synthesis of homocystine (V) reported by Patterson and du Vigneaud (3). The same yields as they report were obtained in preparing homocystine from 0.06 moles of sulfur. However, in the methylation of homocysteine, we were unable to obtain the anticipated yields. It was found that for preparing homocystine, the more convenient reduction of benzylhomocysteine (IV) with sodium and butyl alcohol gave as good yields as reduction with sodium and liquid ammonia. For the preparation of methionine, treatment of the resultant sodium salt of homocysteine with methyl iodide did not give as good yields as reported by Tarver and Schmidt, whether reduction was conducted in liquid ammonia or butyl alcohol. Attempted methylation with dimethyl sulfate and diazomethane did not proceed well.

The method of synthesis of cystine (IX) from

\footnotetext{
1 Part of this work was done in partial fulfillment of the Bachelor's Degree with honors in biochemistry, Harvard University.
}

benzyl mercaptan (I) ( 0.6 moles), reported by Wood and du Vigneaud (4) in 23 per cent yield, was found to give a 21.5 per cent yield when 0.06 mole was used. Reduction of benzylcysteine (VIII) to cysteine with sodium and butyl alcohol did not give good yields; therefore, sodium and liquid ammonia were used. Since radioactive benzyl mercaptan is necessary for the synthesis of all three amino acids, a method of preparation of the mercaptan from hydrogen sulfide, other than that described by Tarver and Schmidt, in 70 per cent yield, was investigated. Elementary sulfur was prepared from hydrogen sulfide by oxidation with iodine, and this was treated with benzylmagnesium chloride. The yield of benzyl mercaptan was 80 per cent. It was found that the yield by the method of Tarver and Schmidt could be raised 4 per cent if the small amount of dibenzylsulfide formed in their reaction was reduced to benzyl mercaptan with sodium in liquid ammonia.

The Fischer synthesis (5) of cystine from $\beta$ chloroaminopropionic acid with barium hydrosulfide was investigated under various conditions. Much poorer yields than the 5 to 10 per cent reported on the basis of chloroaminopropionic acid were obtained when sulfur was not used in large excess.

Attempts to prepare radioactive cystine by replacement reactions of cysteine and radioactive hydrogen sulfide have been described by Tuck (6) and Tarver and Schmidt (2). In a reinvestigation of the problem, no more than 0.3 per cent of available radioactivity was incorporated in cystine (two crystallizations) obtained after heating cysteine hydrochloride with radioactive hydrogen sulfide in aqueous solution at $105^{\circ} \mathrm{C}$. for 10 hours. Heating for longer intervals, at higher temperatures, or at alkaline $\mathrm{pH}$, resulted in destruction of a large fraction of the cysteine.

The enzymatic preparation of cysteine from radioactive hydrogen sulfide has been reported by 


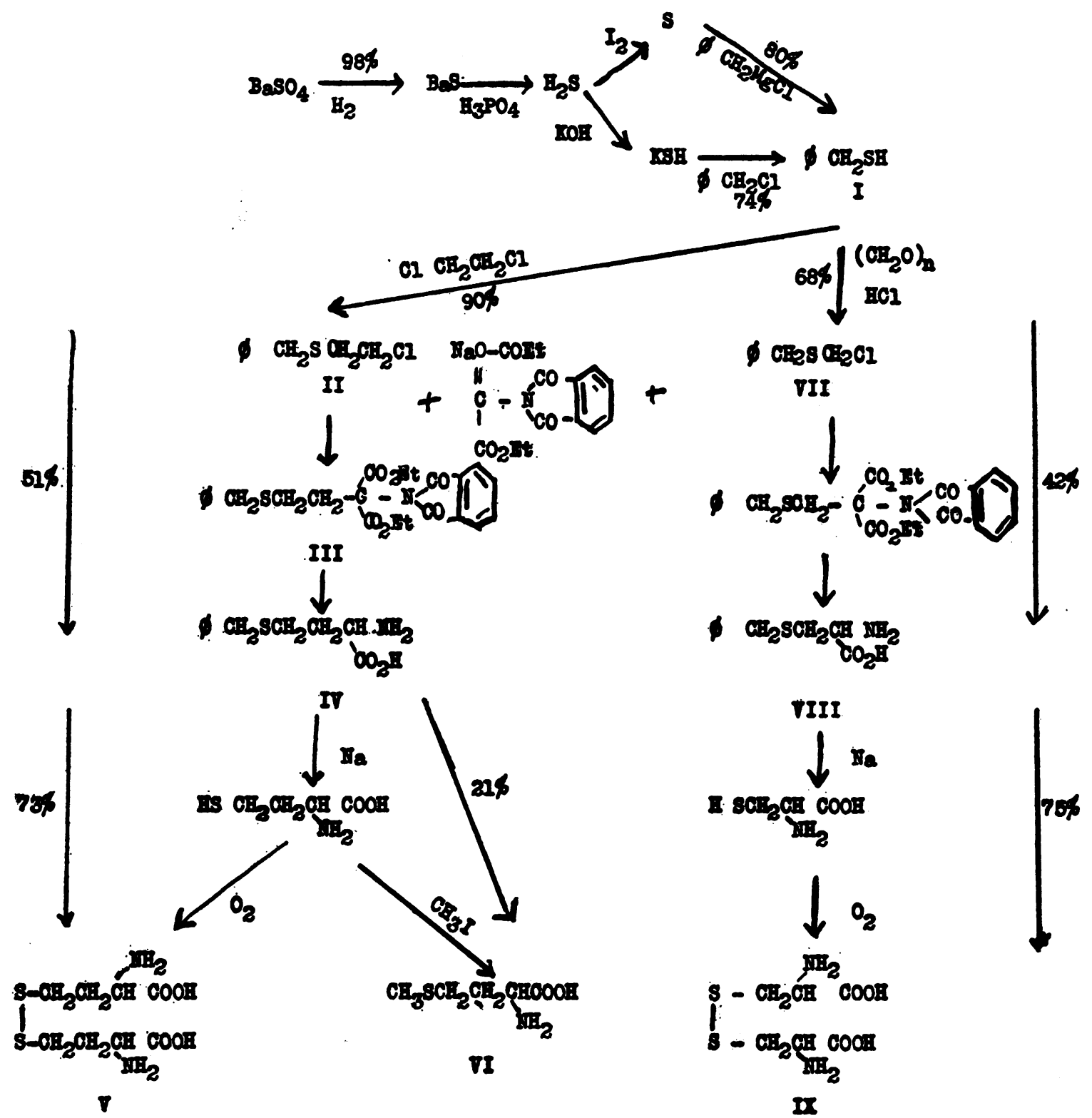

Fig. 1. Scheme for Synthesis of Cystine, Methionine, and Homocystine from Radioactive Sulfur

Smythe and Halliday (7). The isolation of glutathione, from yeast grown on media containing radioactive magnesium sulfate, has been reported by Franklin (8).

\section{EXPERIMENTAL}

Reduction of $\mathrm{BaSO}_{4}$ was accomplished by passing $\mathrm{H}_{2}$ over finely ground barium sulfate at $750^{\circ} \mathrm{C}$. The rate of hydrogen flow was determined by bubbling the gas through concentrated sulfuric acid. A Vicor glass tube, 1 inch in diameter, was heated by means of an encircling electric oven 15 inches in length. The temperature was determined once by means of a thermocouple. Escaping gas was bubbled through ammoniacal cadmium sulfate solution, protected by a trap to eliminate the danger of sucking back. The barium sulfate was spread over the bottom of a porcelain boat, avoiding a layer more than $3 \mathrm{~mm}$. in depth. In a characteristic run, 3.14 grams of barium sulfate were reduced for 5 hours. The loss of weight due to water formed amounted to 85 per cent of the theoretical amount. The sintered barium sulfide was ground to a powder and the reduction continued for another 3 hours. The additional water loss amounted to 5 per cent of the theoretical amount, and the cadmium sul- 
fide formed from the escaping gas amounted to 7.8 per cent; total yield of both sulfides, 97.8 per cent. The oxidation and hydrolysis of barium sulfide was prevented by sealing under glass.

Hydrogen sulfide production occurred more rapidly at the beginning of reduction when water was seen condensing in the cold part of the tube.

Sulfur. Hydrogen sulfide was generated from barium sulfide with 30 per cent phosphoric acid and from cadmium sulfide with concentrated hydrochloric acid. The gas was swept out of the reaction flask with nitrogen and bubbled through two absorption tubes, containing slightly more than an equivalent of iodine dissolved in 20 per cent potassium iodide. The excess iodine was reduced with a little sodium bisulfite and the mixture was neutralized with ammonium hydroxide. The mixture was then heated at $100^{\circ} \mathrm{C}$. for 1 hour to convert the amorphous sulfur to the crystalline form. It was ground to a powder, collected, and washed with water, alcohol, and ether. The yield was quantitative.

Benzyl mercaptan (I) from sulfur. Benzyl magnesium chloride, prepared from 0.12 mole of benzyl chloride and 0.13 mole of magnesium, was allowed to remain in the presence of the excess magnesium overnight to ensure complete reaction of the benzyl chloride. To this was added 2.1 grams (0.065 mole) of sulfur. The mixture was agitated for 24 hours until the sulfur had disappeared. The reaction product was then decomposed with ice and ammonium chloride, extracted with ether, washed, dried, and distilled. Benzyl mercaptan ( 6.5 grams, 80 per cent yield) distilled as a colorless liquid (b.p. $194^{\circ}$ ). The residue, 2.2 grams (b.p. $2 \mathrm{~mm}$. $130^{\circ}$ C.), after reduction with sodium in liquid ammonia failed to give further mercaptan.

Benzyl mercaptan (I) from hydrogen sulfide. Hydrogen sulfide, passed over a small amount of anhydrous calcium chloride, was absorbed in an equivalent of cold $2 \mathrm{~N}$ alcoholic potassium hydroxide ( $0.065 \mathrm{~mole})$. An equivalent of benzyl chloride dissolved in 2 volumes of dry ether was added at once. A fluffy precipitate of potassium hydrosulfide formed, and was gradually replaced by a granular precipitate of potassium chloride on standing in the cold for 15 minutes. The mixture was then heated to the boiling point, after taking the precaution to absorb the hydrogen sulfide which escaped, an additional $2 \mathrm{~N}$ alcoholic potassium hydroxide (10 per cent of the total). This was treated with an additional 10 per cent of the benzyl chloride used, added to the reaction mixture, and allowed to sit several hours. After evaporation of most of the ether, the mixture was used directly in the preparation of benzylthioethyl chloride (II). For the preparation of benzylthiomethyl chloride (III), benzyl mercaptan was isolated. Distillation of the filtrate gave 6 grams ( 74 per cent yield) of benzyl mercaptan (b.p. $194^{\circ}$ ), and 1.5 grams (4.6 per cent yield) of dibenzyl sulfide. Reduction of the latter with sodium and liquid ammonia gave 0.3 gram ( 4 per cent yield) of benzyl mercaptan; total yield 6.3 grams ( 78 per cent). Tarver and Schmidt did not isolate dibenzyl sulfide, and reported a yield of 70 per cent on the basis of the titration of an aliquot.

\section{SYNTHESIS OF HOMOCYSTINE}

Benzylthioethyl chloride (II). To the concentrated reaction mixture containing benzyl mercaptan in 74 per cent yield as described above, was added an equivalent of sodium methylate in the cold. Ten moles of ethylene dichloride (50 grams) were added and the mixture was allowed to stand overnight. It was then heated to the boiling point, cooled, acidified, and washed with water. The washings were extracted with a small amount of ethylene chloride. The combined organic layer was dried with anhydrous sodium sulfate, and the ethylene dichloride distilled at atmospheric pressure. The pressure was lowered and the fraction boiling at 100 to $110^{\circ} \mathrm{C}$. at $3 \mathrm{~mm}$. was taken. The yield was 8 grams ( 90 per cent) of pale yellow oil.

Benzylthioethyl phthalimidomalonic ester (III). The above oil was heated with 18 grams of sodium phthalimidomalonate (9), at $170^{\circ} \mathrm{C}$. for 5 hours. The product was obtained by extraction with toluene.

S-Benzylhomocysteine (IV). The oily product was refluxed with $20 \mathrm{cc}$. alcohol and $35 \mathrm{cc}$. $5 \mathrm{~N} \mathrm{NaOH}$ for 30 minutes. Water (100 cc.) and $20 \mathrm{cc}$. concentrated $\mathrm{HCl}$ were added and refluxing was continued for 30 minutes. After the addition of $50 \mathrm{cc}$. concentrated $\mathrm{HCl}$, refluxing was continued for 1 hour. The solution was then cooled and extracted with ether, and the aqueous portion was evaporated to dryness at reduced pressure. The residue was extracted with boiling absolute alcohol and neutralized with $\mathrm{NH}_{4} \mathrm{OH}$. The precipitate was collected, washed with hot alcohol, and ether. The yield of fine white platelets was 5 grams ( 51 per cent). On the basis of $\mathrm{BaSO}_{4}$, utilizing a 74 per cent yield of benzyl mercaptan, the yield was 33 per cent; that reported by Tarver and Schmidt was 29 per cent.

dl-Homocystine $(V)$. Benzylhomocysteine ( 1.56 grams) was suspended in $30 \mathrm{cc}$. butyl alcohol, and 1.6 grams of sodium were added in small pieces to the refluxing mixture over 2.5 hours. After cooling, the solution was extracted with water, the aqueous extract was neutralized with $\mathrm{HCl}$ and made faintly alkaline with $\mathrm{NH}_{4} \mathrm{OH}$. A crystal of $\mathrm{FeCl}_{3}$ was added, and $\mathrm{O}_{2}$ was bubbled 
into the solution until the color of the dark complex which formed had disappeared. The solution was neutralized, concentrated in vacuo to 75 cc., and allowed to stand overnight. The precipitate was collected, washed with water, alcohol, and ether. The mother liquor was concentrated and yielded additional homocystine; total yield 0.6 gram (73 per cent) of fine white crystals. The yield based on $\mathrm{BaSO}_{4}$ was 24 per cent.

\section{SYNTHESIS OF METHIONINE}

dl-Methionine $(V I)$. Benzylhomocysteine was reduced with sodium and butyl alcohol, as described for the preparation of homocystine. The solution was cooled to $-10^{\circ} \mathrm{C}$. Seven molecular equivalents of methyl iodide were added, and the solution was kept at that temperature for 30 minutes. Water was added; the solution was neutralized and concentrated in vacuo to a small volume. After standing, methionine (as white leaflets) was collected in 21 per cent yield. When more methyl iodide or less was used, and the reaction conducted at a higher temperature, less methionine was recovered. The yield was not improved by following the procedure of Tarver and Schmidt.

The yield on the basis of sulfur used, reported by Tarver and Schmidt, was 19.5 per cent.

\section{SYNTHESIS OF CYSTINE}

Benzylthiomethyl chloride (VII). Following the procedure of Wood and du Vigneaud, using 6.3 grams of benzyl mercaptan, 2.1 grams of polyoxymethylene, 2.5 grams of $\mathrm{CaCl}_{2}$ and dry hydrogen chloride, 6 grams ( 68 per cent yield) benzylthiomethyl chloride was obtained after distillation at water pump pressure (b.p. $130^{\circ}$ ). A crystalline residue boiling about $200^{\circ} \mathrm{C}$. weighed 1.9 grams, probably consisting of di(benzylthio)methane. This same product was obtained in high yield when sodium benzyl mercaptide was treated with an excess of methylene dichloride.

S-Benzylcysteine (VIII). A mixture of 6 grams of benzylthiomethyl chloride, 14. grams of sodium phthalimidomalonic ester, and $30 \mathrm{cc}$. of toluene was refluxed for $2 \frac{1}{2}$ hours, according to the procedure of Wood and du Vigneaud. After filtration and evaporation of the toluene, the viscous oil was hydrolysed according to their pro- cedure. After washing with hot alcohol, the yield was 3.1 grams ( 42 per cent) of fine white platelets.

dl-Cystine (IX). The reduction of 1.25 grams of benzylcysteine with sodium in liquid ammonia and oxidation to cystine, according to the procedure of Wood and du Vigneaud, gave 0.53 grams (75 per cent yield). Overall yield on the basis of $\mathrm{BaSO}_{4}$ was 17 per cent. The yield from benzyl mercaptan was 21.5 per cent; Wood and du Vigneaud obtained a 23 per cent yield. The reduction of benzyl cysteine with sodium and butyl alcohol, as already stated, yielded only traces of cystine.

\section{FISCHER SYNTHESIS}

Bombs containing $\beta$-chloroaminopropionic acid (10) were heated at $100^{\circ} \mathrm{C}$. for several hours with an equivalent of $\mathrm{Ba}(\mathrm{SH})_{2}, \mathrm{KSH}$, and benzyl mercaptan in alcoholic, aqueous and dioxane medium. In no case was cystine or benzylcysteine isolated. The chloroaminopropionic acid was decomposed in each case and the odor of acrylic acid was noted when the tubes were opened.

\section{SULFUR EXCHANGE EXPERIMENT}

In preliminary experiments it was found that cysteine hydrochloride largely decomposed when heated with more than an equivalent of potassium hydrosulfide in a bomb at $100^{\circ} \mathrm{C}$. When sufficient hydrochloric acid was added to the cysteine hydrochloride to neutralize the potassium hydrosulfide, cystine was not recovered after heating for 19 hours at $100^{\circ} \mathrm{C}$. or 1 week at $80^{\circ}$ C. Recovery was 25 per cent after heating 10 hours at $105^{\circ} \mathrm{C}$., 67 per cent after heating 10 hours at $100^{\circ} \mathrm{C}$., and 85 per cent after heating 5 hours at $100^{\circ} \mathrm{C}$.

A tube containing $800 \mathrm{mgm}$. cysteine hydrochloride and 4 drops of concentrated $\mathrm{HCl}$ was suspended in $4 \mathrm{cc}$. water, containing $17 \mathrm{mgm}$. of cadmium sulfide, containing radioactive sulfur. The bomb was sealed and heated with agitation until the cadmium sulfide had dissolved. It was then heated for 5 hours at $100^{\circ} \mathrm{C}$. An 85 per cent recovery of cystine was obtained. Radioactivity measurements indicated incorporation of 0.6 per cent of the radioactive sulfur used. After recrystallization 0.13 per cent incorporation was found. 
In another experiment, using $17 \mathrm{mgm}$. of cadmium sulfide, $1600 \mathrm{mgm}$. of cysteine hydrochloride, 4 drops of concentrated $\mathrm{HCl}$, and heated for 10 hours at $100^{\circ} \mathrm{C}$., 67 per cent recovery of cystine was obtained. Activity measurements indicated incorporation of 2.4 per cent of radioactive sulfur. After recrystallization, 0.26 per cent incorporation was found.

\section{SUM MARY}

Utilizing synthetic methods developed by du Vigneaud and coworkers and by Tarver and Schmidt, three amino acids containing radioactive sulfur were each prepared from 0.06 mole of $\mathrm{BaSO}_{4}$. Cystine was obtained in 17 per cent yield, homocystine in 25 per cent yield. The yield of methionine from homocysteine reported by Tarver and Schmidt was not duplicated.

An improved method for preparing benzyl mercaptan from radioactive sulfur is described.

Radioactive sulfur was generously supplied by $\mathrm{Dr}$. Martin D. Kamen, Radiation Laboratory, Berkeley, California.

The work described in this paper was done under a contract, recommended by the Committee on Medical
Research, between the Office of Scientific Research and Development and Harvard University.

\section{BIBLIOGRAPHY}

1. Kamen, M. D., Production and isotopic assignment of long-lived radioactive sulfur. Physical Rev., 1941, $60,537$.

2. Tarver, H., and Schmidt, C. L. A., Conversion of methionine to cystine; experiments with radioactive sulfur $\left(S^{\$ 5}\right)$. J. Biol. Chem., 1939, 130, 67.

3. Patterson, W. I., and du Vigneaud, V., The synthesis of homocystine. J. Biol. Chem., 1935, 111, 393.

4. Wood, J., and du Vigneaud, V., A new synthesis of cystine. J. Biol. Chem., 1939, 131, 267.

5. Fischer, E., and Raske, K., Verwandlung des 1Serins in aktives natürliches Cystin. Ber. d. chem. Gesellsch., 1908, 41, 893.

6. Tuck, J. L., Radioactive sulfur for biochemical experiments. J. Chem. Soc. (London), 1939, 1292.

7. Smythe, C. V., and Halliday, D., An enzymatic sulfur exchange between cysteine and radioactive sulfides. Federation Proceedings, 1942, 1, 16.

8. Franklin, R. G., The preparation of glutathione containing radioactive sulfur. Science, 1939, 89, 298.

9. Organic Syntheses, 1927, Vol. VII, 78.

10. Fischer, E., and Raske, K., Verwandlung des 1-Serins in d-Alanin. Ber. d. chem. Gesellsch., 1907, 40, 3717.

11. Seligman, A. M., A modified electroscope especially suited for measuring substances with low energy radiation. J. Clin. Invest., 1943, 22, 281. 